\title{
Zellkulturtechnik
}

\section{Aktuelle Trends bei der in vitro-Substanz- testung in Deutschland und der Schweiz}

\author{
RALF PÖRTNER ${ }^{1}$, ILKA WAGNER ${ }^{2}$, TAMARA ATANASOVA ${ }^{2}$, RICHA GAUTAM ${ }^{1}$, JASMIN GATTLEN ${ }^{3}$, MARKUS RIMANN ${ }^{3}$, \\ EPIFANIA BONO ${ }^{3}$, URSULA GRAF-HAUSNER ${ }^{3}, \mathrm{UWE} \mathrm{MARX}^{2}$ \\ ${ }^{1}$ INSTITUT FÜR BIOPROZESS- UND BIOSYSTEMTECHNIK, TU HAMBURG-HARBURG \\ 2 MEDIZINISCHE BIOTECHNOLOGIE, TU BERLIN \\ ${ }^{3}$ KOMPETENZZENTRUM TEDD, ZÜRCHER HOCHSCHULE FÜR ANGEWANDTE WISSENSCHAFTEN WÄDENSWIL, SCHWEIZ
}

DOI: $10.1007 / \mathrm{s} 12268-014-0466-6$

(C) Springer-Verlag 2014

Der Bedarf an Testsystemen für chemische und pharmakologisch aktive Substanzen ist aufgrund regulatorischer Vorgaben (EU-Chemikalienverordnung REACH, Kosmetikverordnung) immens gestiegen. Anstelle der heute noch üblichen Tierversuche sollen künftig organotypische Gewebekulturen treten, deren

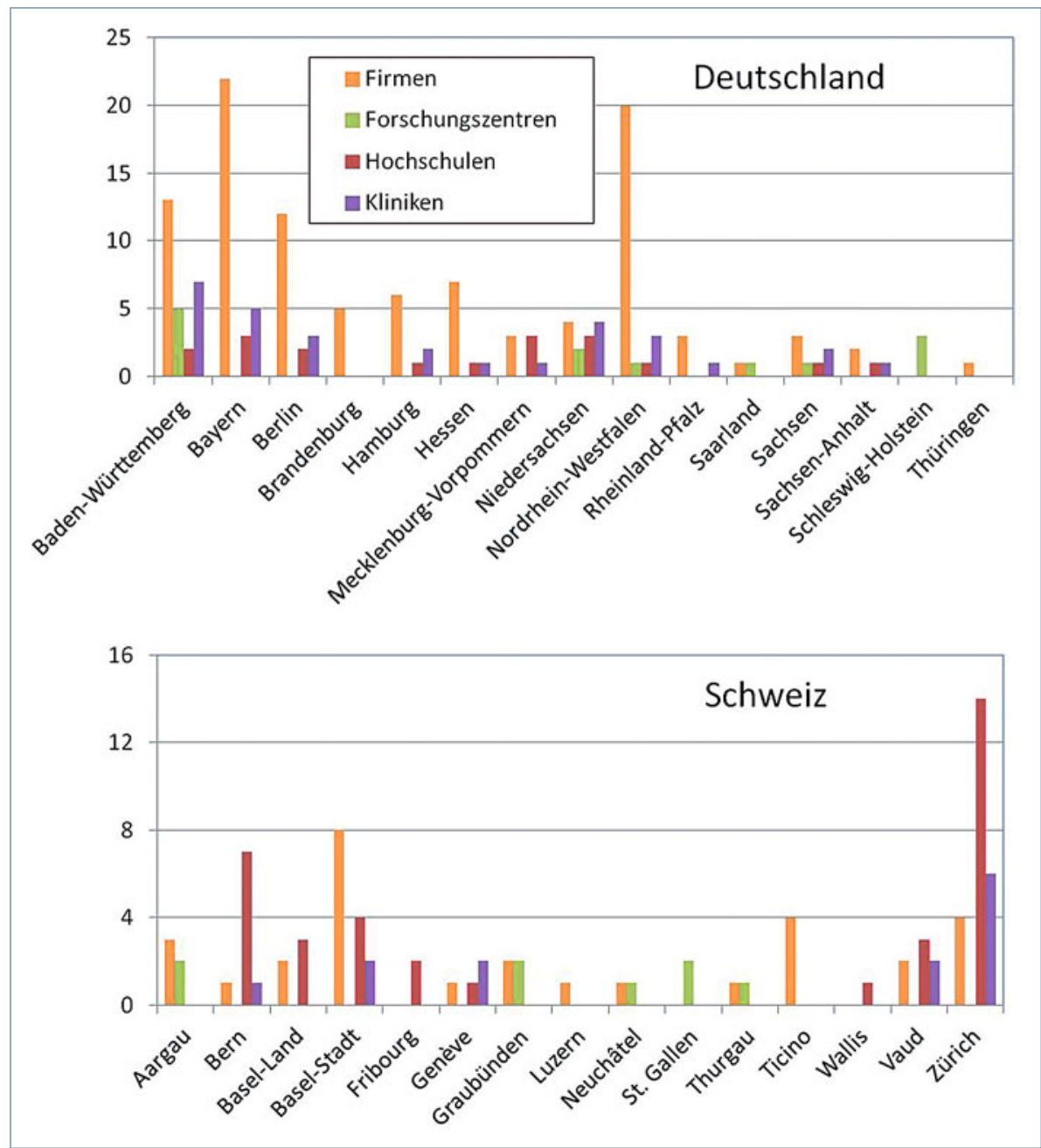

$\Delta$ Abb. 1: Verteilung der Institutionen, die an der Umfrage teilgenommen haben, auf die Bundesländer (Deutschland) und Kantone (Schweiz). Nicht genannte Bundesländer bzw. Kantone blieben ohne Antwort.

Aussagekraft eine höhere Relevanz verspricht. Tierversuche werfen zur ethischen Problematik weitere Limitierungen wie die unzureichende Verfügbarkeit oder die häufig nicht gewährleistete Übertragbarkeit von Daten aus dem Tiermodell auf den Menschen auf. Es wird erwartet, dass durch organotypische Gewebekulturen die Medikamentenentwicklung und Wirkstofftestung sicherer und vorhersehbarer werden. Die neuen Testverfahren sind des Weiteren durch die Verwendung von patientenspezifischem Material als Ergänzung zu klinischen Studien zu sehen, da dadurch die genetische Vielfalt der Patienten besser berücksichtigt werden kann.

Nach einer DECHEMA-Umfrage im Jahr 2009 zu akademischen und industriellen Forschungsaktivitäten auf dem Gebiet der Zellund Gewebekulturtechnik für die regenerative Medizin und Substanzprüfungen in Deutschland [1] wurde im Jahr 2013 eine Neubewertung der Aktivitäten in diesem Bereich durchgeführt und um die Aktivitäten in der Schweiz erweitert. Die Umfrage wurde zudem stärker auf die in vitro-Substanztestung einschließlich der Nutzung systembiologischer Methoden ausgerichtet. Auf deutscher Seite wurde die Studie durch die Beiräte der Fachgruppen „Zellkulturtechnologie“ und „Medizinische Biotechnologie" der DECHEMA e. V., auf schweizerischer Seite vom nationalen Kompetenzzentrum TEDD (Tissue Engineering for Drug Development and Substance Testing) getragen.

\section{Methodik der Studie}

Zunächst wurden die wichtigsten deutschen und schweizerischen Institutionen aus Akademie und Industrie auf dem Gebiet „Substanztestung und regenerative Medizin“ ermittelt. Dazu wurden Erhebungen in Online-Datenbanken zu Veröffentlichungen, 
Patenten und staatlichen Förderprogrammen nach vorgegebenen Stichwörtern, außerdem Erhebungen in den Datenbanken des nationalen thematischen Netzwerks Swiss Biotech genutzt. Des Weiteren diente die Analyse von Veröffentlichungen, Kongressen und Messen neben anderen (beim Korrespondenzautor einsehbaren) Quellen der Ermittlung von geeigneten Institutionen.

An diese ausgewählten Institutionen wurde ein Fragebogen verschickt, der folgende Aspekte umfasste:

- Werden in vitro Zellkultursysteme für die Substanztestung und/oder regenerative Medizin verwendet?

- Werden Omics-Techniken (Genomics, Transcriptomics, Metabolomics, Proteomics oder sonstige) für die Zellkulturanalyse verwendet?

- Werden Omics-Datensätze in der systembiologischen Auswertung zusammengeführt?

- Welche Arten von Zellquellen (primäre Zellen, Gewebe bzw. Organe oder Zelllinien) werden genutzt?

- Werden in Testansätzen mehrere unterschiedliche organotypische Kulturen miteinander kombiniert, um Organwechselwirkungen nachzubilden?

\section{Ergebnisse}

Für Deutschland ergab die Recherche 1.146 Kontakte an akademischen Institutionen, von denen 62 den Fragebogen beantworteten. Die Suche nach Firmen führte zu 141 Kontakten bei 103 positiven Antworten. Für die Schweiz wurden 104 akademische Gruppen und 228 Firmen identifiziert, mit 56 bzw. 30 positiven Antworten.

Bei den akademischen Institutionen in Deutschland fielen mehr als 30 Kontakte auf die Regionen Aachen (38), Berlin (82), Dresden (37), Freiburg (39), Hannover (57), Heidelberg (70), Leipzig (35), München (85), Münster (31), Regensburg (30), Tübingen (44), Würzburg (34). Dies spiegelt im Wesentlichen die Standorte der großen klinischen Zentren wider. Die Firmen in Deutschland finden sich zum überwiegenden Teil in den Bundesländern Baden-Württemberg, Bayern, Berlin und Nordrhein-Westfalen (Abb. 1). Für die Schweiz konzentrieren sich die akademischen Institutionen auf die Kantone Bern, Basel und Zürich, die Firmen auf die Kantone Basel, Ticino und Zürich (Abb. 1).

Das Ergebnis der ausgewerteten Fragebögen erlaubt für Deutschland folgende Schlüsse (Abb. 2):

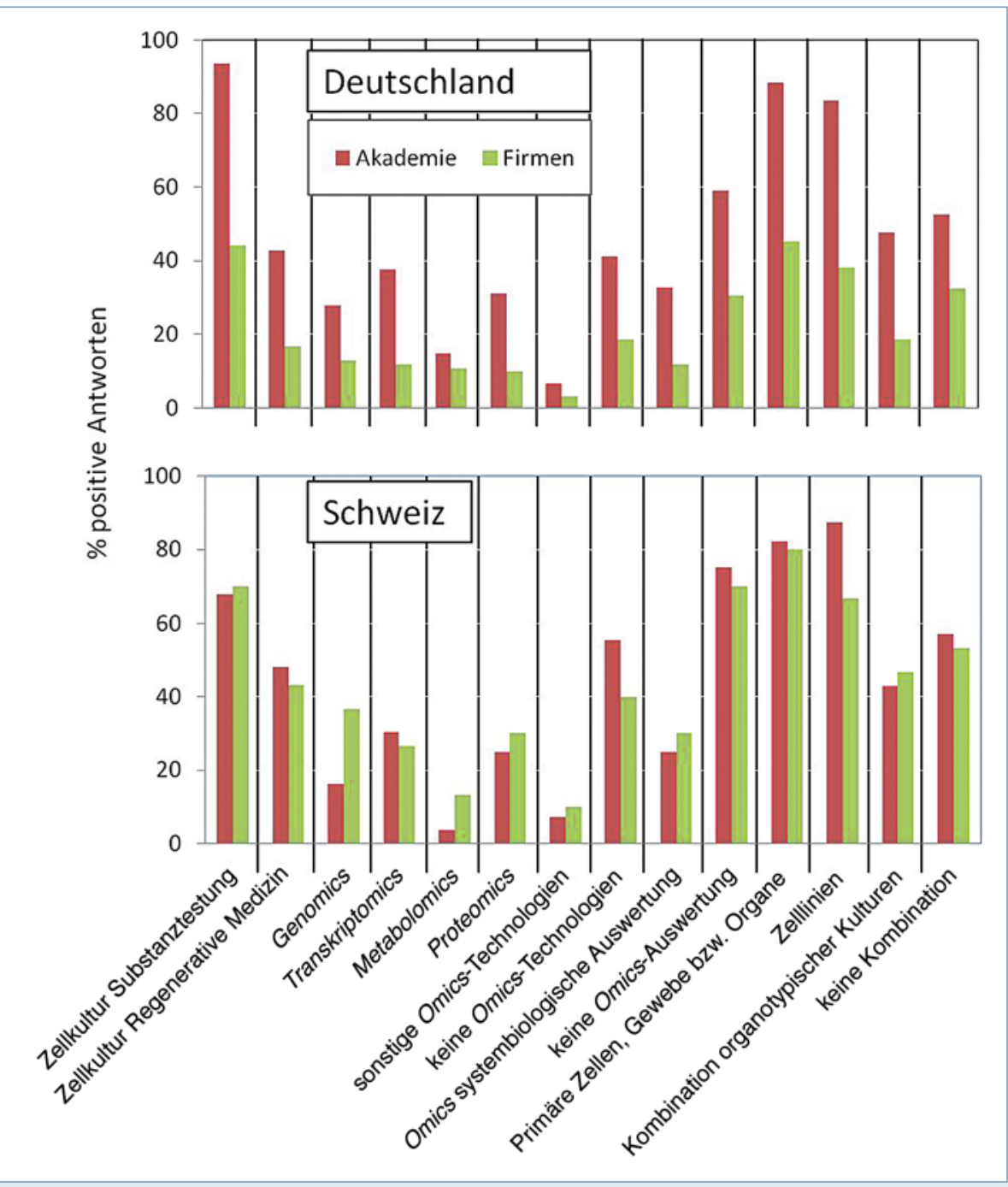

$\Delta$ Abb. 2: Einsatz von verschiedenen Methoden der in vitro-Substanztestung bei akademischen Institutionen und Firmen in Deutschland und der Schweiz.

- Es sind mehr akademische Institutionen und Firmen in der „Substanztestung“ aktiv als in der „regenerativen Medizin“. Hier ist allerdings zu berücksichtigen, dass die Rücklaufquote bei akademischen Institutionen gering war. Es ist nicht auszuschließen, dass viele der akademischen Institutionen, die nicht geantwortet haben, sich von „Substanztestung“ nicht angesprochen fühlten.

- Die relative Anzahl der Firmen, die sowohl in der „Substanztestung“ als auch in der „regenerativen Medizin“ aktiv sind, ist gering.

- Omics-Techniken werden anscheinend vor allem in akademischen Institutionen angewendet.

- Gleiches gilt für primäre Zellen, Gewebe und Organe sowie für organotypische Gewebekulturen.
Für die Schweiz ergibt sich Folgendes (Abb. 2):

- In der Relation zu Deutschland sind mehr akademische Institutionen und Firmen in „regenerativer Medizin“ aktiv.

- Auch die relative Anzahl der Firmen, die sowohl in der „Substanztestung“ als auch in der „regenerativen Medizin“ aktiv sind, scheint höher zu sein.

- Omics-Techniken sind offenbar gut in der Industrie etabliert, weniger in der Akademie.

- Primäre Zellen, Gewebe und Organe sowie organotypische Gewebekulturen scheinen in der Akademie und bei Firmen gut etabliert zu sein.

\section{Diskussion und Schlussfolgerungen}

Für Deutschland lässt sich aus den aktuellen Daten im Vergleich zur Umfrage 2009 [1] 
grundsätzlich eine erhebliche Zunahme der Aktivitäten im Bereich „regenerative Medizin/Substanztestung" registrieren. So wurden damals nur 161 akademische Institutionen identifiziert, bei der aktuellen Umfrage hingegen über 1.000. Bei den identifizierten Firmen hat sich die Anzahl etwa verdoppelt, der überwiegende Teil im Bereich der „Substanztestung“. Im Jahr 2009 wurden aber noch mehr Firmen im Bereich „regenerative Medizin“ identifiziert. Die Überlappung beider Bereiche ist demnach bei Firmen gering. Daraus ist zu schließen, dass die industrielle „Substanztestung“ an Bedeutung erheblich zugenommen hat. Dabei scheint bei aktuellen Methoden wie den Omics-Techniken oder der Nutzung von Geweben oder organotypischen Strukturen noch Nachholbedarf zu bestehen.

Im Vergleich dazu konnte für die Schweiz kein wesentlicher Unterschied zwischen „Substanztestung“ und "regenerativer Medizin“ gefunden werden, beide Bereiche sind hier offenbar gleich bedeutend. Auch in Bezug auf die Anwendung von Omics-Techniken zeigen sich Unterschiede: In der Schweiz sind diese anscheinend sehr viel besser in der Industrie etabliert. Außerdem werden organotypische Gewebekulturen hier auch industriell eingesetzt.

Insgesamt unterstreicht die Studie die zunehmende Bedeutung von zellbasierter Substanztestung, auch im industriellen Umfeld. Dabei ist das Potenzial neuer Technologien (Omics, organotypische Kulturen) bei Weitem noch nicht ausgeschöpft. Da die Einführung dieser Technologien üblicherweise sehr zeit- und kostenintensiv ist und zudem eine enge Kollaboration verschiedener Fachgebiete in Wissenschaft und Ingenieurwesen bedingt, sollten nationale und transnationale Fördermaßnahmen hier unterstützend eingesetzt werden. In Anbetracht der weit verbreiteten grundsätzlichen Vorbehalte gegenüber (schwer)belastenden Tierversuchen kommt zudem der Förderung alternativer in vitroTestmethoden eine außerordentlich wichtige gesellschaftliche Bedeutung zu.

\section{Danksagung}

Wir bedanken uns herzlich für die finanzielle Unterstützung der Studie durch die DECHEMA und die Gebert Rüf Foundation.

\section{Literatur}

[1] Pörtner R, Burger C, Maier-Reif K et al. (2010) Organotypic tissue cultures for substance testing - a survey of academic and industrial activities in Germany. BIOforum Europe 3:21-23

\section{Korrespondenzadresse:}

Prof. Dr.-Ing. habil. Ralf Pörtner Technische Universität HamburgHarburg

Institut für Bioprozess- und Biosys-

temtechnik

Denickestraße 15

D-21073 Hamburg

Tel.: 040-42878-2886

Fax: 040-42878-2909

poertner@tuhh.de 